curity sector reform, in Iraq and elsewhere, is the security of the population and, only as a means to that end, the security of the state.

How then to best take advantage of the complementarity between the two concepts?

Three considerations can be put forward here. The first is that for the academic and policy communities that are involved in human security and security sector reform, there are good reasons for accepting the proposition that while different in a number of respects, the two concepts enjoy important conceptual links. Secondly, it should be acknowledged that their complementarity can also be useful in practical terms. Human security can help guide practitioners of security sector reform, while security sector reform can help operationalize the practice of human security. Thirdly, those who design and implement programmes around human security themes or who support the reform of security sectors should be encouraged to bring together elements from the two concepts and their experience in the field into their own work. Moreover, in view of the many issues that are addressed by both human security and security sector reform, joint human security-security sector reform programs should certainly be feasible. The Human Security Network could be a particularly promising framework for developing programmes that take advantage of this complementarity and seek out synergies.

The bottom line in all this is that without a functional security sector, the state will not be able to provide the secure environment that is required to realize human security goals. Similarly, unless guided by a human security perspective, security sector reform risks generating a security sector that is not accountable to those it is supposed to serve and that can act oppressively towards them. These are terribly real problems in today's world, where there are far too frequent instances of states failing to meet their security responsibilities - whether this is because of resource shortages, inefficiency, neglect or ill will towards the people in their care.

\title{
Human Security als Teil einer geschlechtersensiblen Außen- und Sicherheitspolitik?
}

\author{
Cornelia Ulbert*
}

\begin{abstract}
Human security is a highly controversial concept. Whilst proponents praise it for its focus on individuals and its practical, i.e. normative relevance, critics complain about the broadness and fuzziness of the concept. In this article, the author makes the case for a narrower concept of human security from a gender perspective. A comparison of the concept of human security with the traditional concept of state security and a broader notion of security that was developed after the end of the Cold War proves the potential of human security to also encompass questions of gender difference and gender equity. However, subsuming development and human rights issues under the heading of human security risks losing the empowerment and rights dimensions of the other perspectives. Therefore, a narrower conception of human security, and one that focuses on the physical and psychological integrity of individuals would seem to be more appropriate.
\end{abstract}

Keywords: Menschliche Sicherheit, Geschlecht und Sicherheit, menschliche Sicherheit als Außenpolitikstrategie.

$\mathrm{Z}$ hn Jahre, nachdem das Konzept von Human Security in einem der Human Development Reports des UNEntwicklungsprogramms UNDP einer breiteren Öffentlichkeit vorgestellt wurde, ${ }^{1}$ hat der Begriff mittlerweile Einzug in die breitere wissenschaftliche und politische Debatte gefunden. Dies verdankt er auch einem Bericht, den die Commission on Human Security unter dem appellativen Titel Human Security Now im Mai 2003 veröffentlichte. ${ }^{2}$ Dieser Be-

\footnotetext{
* Dr. Cornelia Ulbert ist wissenschaftliche Mitarbeiterin an der Arbeitsstelle für Transatlantische Außen- und Sicherheitspolitik des Otto-SuhrInstituts, Freie Universität Berlin.

1 United Nations Development Programme, Human Development Report 1994, New York/Oxford 1994 (im Internet abrufbar unter: http://hdr.undp.org/reports/global/1994/en/).

2 Commission on Human Security, Human Security Now, New York 2003
} (http://www.humansecurity-chs.org/finalreport/index.html). richt ist das Ergebnis eines etwa zweijährigen Diskussionsprozesses, der mit der Einsetzung der Commission on Human Security im Januar 2001 begann. ${ }^{3}$ Diese Kommission wurde auf Initiative Japans im Anschluss an den so genannten Millenniumsgipfel ${ }^{4}$ der Vereinten Nationen vom September 2000 unter Vorsitz der ehemaligen UN-Hochkommissarin für Flüchtlinge Sadako Ogata und des Nobelpreisträgers für Wirtschaft Amartya Sen ins Leben gerufen. Auslöser war die Erkenntnis, dass nicht nur bewaffnete Konflikte, sondern auch Armut, Hunger, Infektionserkrankungen oder auch Menschenrechtsverletzungen das Leben und die Sicherheit

\footnotetext{
3 Nähere Informationen zur Arbeit der Kommission finden sich unter http://www.humansecurity-chs.org.

4 Zum Millenniumsgipfel der Vereinten Nationen siehe http://www.un.org/millennium.
} 
von Menschen negativ beeinflussen. Ziel der Kommissionsarbeit sollte es sein, Konzepte und Lösungsvorschläge zu entwickeln, wie das zweifache Ziel gewährleistet werden könnte, das UN-Generalsekretär Kofi Annan beim Millenniumsgipfel formuliert hatte, nämlich Menschen ein Leben in freedom from want und freedom from fear, also in Freiheit von Mangel und Angst zu sichern.

Einerseits wird menschliche Sicherheit enthusiastisch als Konzept gefeiert, in dem Menschen im Mittelpunkt stehen, ${ }^{5}$ andererseits weisen KritikerInnen gleichzeitig darauf hin, dass das Konzept eher ein shotgun approach, ${ }^{6}$ also die berühmte Lösung mit der Schrotflinte sei, wenn es darum ginge, menschliches Leid zu mindern. Auf theoretischkonzeptioneller Ebene wird heftig um die Frage gerungen, ob das Konzept von menschlicher Sicherheit zu breit angelegt sei und nicht klarer und damit gleichzeitig enger definiert werden solle. Gleichzeitig wird die praktisch-politische Relevanz des Konzepts hervorgehoben, die auf seinen normativen Gehalt zurückzuführen sei. ${ }^{7}$ Fasst man die Diskussion um Human Security zusammen, so scheint die Konzentration des Konzepts auf Individuen Zustimmung zu finden, dessen praktische Umsetzbarkeit jedoch eher bezweifelt $z u$ werden.

Legt man diese widersprüchlichen Bewertungen zugrunde, stellt sich die Frage, ob das Konzept von menschlicher Sicherheit die Erwartungen, die gerade aus feministischer oder geschlechterpolitischer Perspektive damit verbunden werden, tatsächlich erfüllen kann. Um diese Frage zu beantworten, werde ich zunächst den traditionellen Sicherheitsbegriff im Vergleich mit dem erweiterten Sicherheitsbegriff, der sich vor allem seit dem Ende des Ost-West-Konflikts entwickelt hat, und dem Konzept von menschlicher Sicherheit daraufhin untersuchen, was oder wer aus der Perspektive der unterschiedlichen Sicherheitsbegriffe mit welchen Mitteln vor welchen Gefährdungen geschützt werden soll. Darauf aufbauend werde ich argumentieren, dass der mit dem Konzept von menschlicher Sicherheit vorgenommene Paradigmenwechsel hin zu Individuen im Gegensatz zu den anderen Sicherheitsbegriffen eine geschlechterorientierte Perspektive ermöglicht, aus konzeptionellen und praktisch-politischen Gründen jedoch eine deutliche Verengung des Begriffs von menschlicher Sicherheit auf die körperliche und geistige Unversehrtheit von Individuen notwendig ist.

\section{Konkurrierende Sicherheitsbegriffe}

Mit Auflösung des Ost-West-Konflikts zu Beginn der 1990er Jahre setzte verstärkt eine Debatte um die Erweiterung des Si-

5 Lloyd Axworthy, »Human Security and Global Governance: Putting People First «, in: Global Governance (1) 2001, S. 19-23.

6 Yuen Foong Khong, »Human Security: A Shotgun Approach to Alleviating Human Misery? «, in: Global Governance (3) 2001, S. 231-236.

7 Die gesamte Bannbreite der Debatte um Human Security wird sehr schön in einer »Special Section « der Zeitschrift »Security Dialogue «um Thema "What is ,Human Security' « deutlich, in der 21 AutorInnen gebeten wurden, ihre Einschätzungen zu diesem Konzept und zu dessen Relevanz abzugeben (vgl. Security Dialogue [3] 2004, S. 345-371). cherheitsbegriffs ein. ${ }^{8}$ Auffällig daran war allerdings, dass zunächst vor allem traditionelle Sicherheitsforscher und Militärs auf der Basis eines (neo)realistischen Verständnisses von staatlicher Sicherheit darum bemüht waren, vor neuen Sicherheitsgefahren zu warnen. Verständlicherweise stand dahinter auch der Versuch, die jeweiligen finanziellen Ausstattungen in Zeiten fehlender Blockkonfrontation zu sichern. Symptomatisch hierfür ist die Debatte um »Umwelt und Sicherheit «, die vor allem in der US-amerikanischen, am politischen Realismus orientierten sicherheitspolitischen Zeitschrift International Security geführt wurde. ${ }^{9}$ Die Gegenreaktion derjenigen, die sich traditionell mit Entwicklungsfragen beschäftigt haben, ließ jedoch nicht lange auf sich warten. Mit einer Ausweitung des Sicherheitsbegriffs auf menschliche Sicherheit forderten auch sie einen Teil der so genannten Friedensdividende für entwicklungsrelevante Projekte ein. So stand der Begriff Human Security im oben erwähnten Human Development Report von 1994 im Mittelpunkt der Argumentation zur Verbesserung menschlicher Entwicklungschancen. Eine Reihe von Staaten ${ }^{10}$ gründeten im Anschluss an die erfolgreiche Kampagne zum Bann von Antipersonen-Landminen, die zur Ottawa-Konvention führte, 1999 das Human Security Network, ${ }^{11}$ um in ihren außenpolitischen Strategien dem Gedanken der menschlichen Sicherheit Rechnung $\mathrm{zu}$ tragen. $\mathrm{Zu}$ den international bekanntesten staatlichen Förderern des Konzepts - wiewohl mit unterschiedlicher Akzentsetzung - gehören Japan und Kanada. ${ }^{12}$ Vor dem Hintergrund dieses Kontextes werden die Intentionen, die sich mit diesem Begriff verbinden, deutlich: Human Security dient zum einen als Mobilisierungsbegriff für eine sehr heterogene Koalition von humanitären und entwicklungspolitisch engagierten NGOs, internationalen Organisationen und so genannten Mittelmächten, ${ }^{13}$ zum anderen soll damit bestimmten Themen eine höhere Priorität auf der politischen Tagesordnung zugewiesen werden. Doch ist das Konzept so breit, dass sich die Frage stellt, inwiefern sich die Hoffnungen, die sich mit menschlicher Sicherheit - auch aus einer geschlechterpolitischen Perspektive - verbinden, tatsächlich realisieren lassen.

8 Einen guten Überblick über die Debatte geben Keith Krause/Michael C. Williams, „Broadening the Agenda of Security Studies: Politics and Methods«, in: Mershon International Studies Review (2) 1996, S. 229-254.

9 Zu der kontrovers geführten Debatte in International Security vgl. vor allem die Beiträge von Thomas F. Homer-Dixon, »On the Threshold: Environmental Changes as Causes of Acute Conflict «, in: International Security (2) 1991, S. 76-116, Thomas F. Homer-Dixon, »Environmental Scarcities and Violent Conflict: Evidence from Cases", in: International Security (1) 1994, S. 5-40, Thomas F. Homer-Dixon/ Marc A Levy, »Environment and Security", in: International Security (3) 1995, S. 189-198; Marc A. Levy, "Is the Environment a National Security Issue? «, in: International Security (2) 1995, S. 35-62. Zur Kritik aus der Perspektive der Friedens- und Konfliktforschung vgl. Nils Petter Gleditsch, "Armed Conflict and the Environment: A Critique of the Literature«, in: Journal of Peace Research (3) 1998, S. 381-400, Nina Graeger, "Environmental Security? «, in: Journal of Peace Research (1) 1996, S. 109-116. Als allgemeinen Überblick über die Forschung zu »Umwelt und Sicherheit « siehe Carsten F. Ronnfeldt, »Three Generations of Environment and Security Research «, in: Journal of Peace Research (4) 1997, S. 473-482.

10 Dazu zählen Chile, Griechenland, Irland, Jordanien, Kanada, Mali, die Niederlande, Norwegen, Österreich, die Schweiz, Slowenien, Thailand und als Beobachter Südafrika.

11 Vgl. http://www.humansecuritynetwork.org.

12 Vgl. die entsprechenden Websites des japanischen Außenministeriums (http://www.mofa.go.jp/policy/human_secu/index.html) und des kanadischen Außenministeriums (http://www.humansecurity.gc.ca).

13 Vgl. auch Roland Paris, »Human Security: Paradigm Shift or Hot Air? «, in: International Security (2) 2001, S. 87-102, hier S. 88. 
Der traditionelle Sicherheitsbegriff beschränkt sich auf die nationale Sicherheit, das heißt auf den Schutz eines staatlichen Territoriums. Vergleicht man diesen mit dem erweiterten Sicherheitsbegriff, wie er auch der deutschen Außenpolitik zugrunde liegt, zeigt sich, dass zunächst nur das Spektrum der Gegenstände ausgeweitet wird, die als mögliche Bedrohung für staatliche Sicherheit verstanden werden. Neu in den Blick rücken Bedrohungen für die Umwelt oder die Wirtschaft, innerstaatliche Konflikte oder der internationale Terrorismus. Im Mittelpunkt stand und steht jedoch auch beim erweiterten Sicherheitsbegriff der Schutz des Staates vor äußeren und inneren Gefährdungen. Die Quelle der Bedrohung mag sich vom klassischen Sicherheitsdilemma hin $\mathrm{zu}$ »neuen Kriegen « verschoben haben, die nach Auflösung der bipolaren Strukturen Kennzeichen alter oder neuer Konflikte in vielen Regionen der Welt waren und sind. Hinzu kommen grenzüberschreitende Problemlagen wie Menschenhandel, transnational organisierte Kriminalität und internationaler Terrorismus, auf die jedoch weiterhin mit dem Einsatz staatlicher Gewalt - und dies heißt nicht zuletzt militärischer Gewalt - reagiert wird. Unter dem erweiterten Sicherheitsbegriff sind Staaten bemüht, ihre staatliche Gewalt den veränderten Bedrohungsszenarien anzupassen, indem sie beispielsweise die internationale Zusammenarbeit mit anderen Staaten intensivieren. Allerdings ermöglicht auch der erweiterte Sicherheitsbegriff aufgrund seiner Staatszentriertheit keine befriedigende Integration der Geschlechterperspektive.

Das Konzept von menschlicher Sicherheit zeigt demgegenüber durch den radikalen Wechsel hin zur Perspektive des Individuums größere Nähe zu den Forderungen, die von Seiten feministischer und gender-orientierter Forschungen immer wieder gestellt wurden. ${ }^{14}$ So hatte Ann Tickner beispielsweise vor Jahren in ihrer Kritik am realistischen Sicherheitsbegriff darauf hingewiesen, dass die Trennlinie zwischen Anarchie und Ordnung nicht entlang der Trennlinie zwischen internationalem und nationalem System verlaufe. ${ }^{15}$ Das Phänomen häuslicher Gewalt und die Betroffenheit von Frauen und Kindern durch Krieg zeigt deutlich, dass die »weibliche « Perspektive auf Gewalt ein anderes Bild vermittelt, als das eines internationalen anarchischen Selbsthilfesystems auf der einen und die durch das staatliche Gewaltmonopol zivilisierte Sphäre des Nationalstaates auf der anderen Seite. Aus einer Gender-Perspektive, die die Konstruktion von Geschlechterrollen und -stereotypen sowie die Ungleichheit der Geschlechter und die daraus resultierenden asymmetrischen Machtverhältnisse in den Blick nimmt, ist der Staat damit nicht unbedingt als Garant von Sicherheit zu verstehen. Vielfach erweist sich das Ringen um Nationalstaatlichkeit und bestimmte Formen der Herausbildung staatlicher Identität geradezu als Problem für

14 Vgl. hierzu V. Spike Peterson (Hrsg.), „Gendered States. Feminist (Re)Visions of International Relations Theory", Boulder/London 1992, J. Ann Tickner, »Gender in International Relations. Feminist Perspectives on Achieving Global Security«, New York 1992, Ann Tickner, »Re-visioning Security «, in: Ken Booth/Steve Smith (Hrsg.): International Relations Theory Today, Cambridge 1995, S. 175-197, J. Ann Tickner, Gendering World Politics: Issues and Approaches in the Post-Cold War Era, New York 2001.

15 J. Ann Tickner, "Hans Morgenthau's Principles of Political Realism: A Feminist Reformulation «, in: Millennium (3) 1988, S. 429-440. einzelne schwache Gruppen einer Gesellschaft. Die aus einer Geschlechterperspektive geäußerte Kritik an der Trennung von außen und innen, die analog der Trennung zwischen öffentlich und privat zu sehen ist, ging auch einher mit einer Kritik am klassischen realistischen Verständnis von »Bürgertugenden «. Nach feministischem Verständnis liegen diese nicht nur auf militärischem Gebiet (warrior-patriot), das traditionellerweise eine höhere Wertschätzung erhält, auch soziale Dienste zeugen von "gutem « staatsbürgerlichem Verhalten (citizen-defender). Daraus lassen sich andere, vor allem zivile Formen der Konfliktbearbeitung ableiten und begründen, die basierend auf einem traditionellen Verständnis von staatlicher Sicherheit nicht in Betracht gezogen werden. Auch ein feministisches Verständnis von Macht, das diese nicht mehr als Kontrolle über (power over) definiert, sondern als Bündelung von Kapazitäten, um ein bestimmtes Ziel zu erreichen (power to), lässt sich eher mit dem Konzept von Human Security vereinbaren als mit dem klassischen oder erweiterten Sicherheitsbegriff. Denn Human Security richtet das Augenmerk auf die »menschlichen Aspekte von Sicherheit, Rechten und Entwicklung, weshalb auch Post-Konflikt-Situationen, Armut oder Gesundheitsgefährdungen thematisiert werden. Als Quelle der Bedrohungen werden politische und ökonomische Ungleichheiten angesehen, die auf die Auswirkungen von Globalisierungsprozessen zurückgeführt werden. Während beim traditionellen und beim erweiterten Sicherheitsbegriff der Einsatz staatlicher Gewalt zur Abwehr möglicher Bedrohungen im Vordergrund steht, zeigt das Konzept von menschlicher Sicherheit eine Reihe von - vor allem zivilen - Bearbeitungsstrategien auf, in die eine Vielzahl von Akteuren eingebunden sind. Neben Staaten sind dies sowohl internationale Organisa-tionen, als auch zivilgesellschaftliche und privatwirtschaftliche Akteure. Zudem ist dabei nicht allein der Schutz, sondern auch das Empowerment der Betroffenen die Zielperspektive (siehe Schaubild 1).

Obwohl es insbesondere aus einer Geschlechterperspektive begrüßenswert ist, dass nun der Sicherheitsfokus auf Individuen und nicht auf Staaten liegt, so kann sich die Umetikettierung traditioneller entwicklungsrelevanter und rechtlicher Themen zu Sicherheitsthemen auch als Pyrrhussieg erweisen. Mit der $»$ Versicherheitlichung ${ }^{16}{ }^{16}$ einzelner Bereiche ändert sich nämlich zweierlei: Erstens ändert sich die Art und Weise, wie die Situation wahrgenommen wird. Wenn jemand oder etwas bedroht wird, entsteht eine Abwehrhaltung, denn derjenige, der mein Wohlergehen bedroht, wird zu meinem »Feind «. Zweitens ändert sich damit auch das Repertoire der möglichen Problembearbeitung hin zu einer militärischen Perspektive. Das Konzept der so genannten humanitären Intervention beispielsweise führt deutlich die Folgen der Ausweitung des Sicherheitsbegriffs vor Augen, da nunmehr Menschenrechte auch mit militärischen Mitteln geschützt werden sollen. In der Debatte um »Umwelt und Sicherheit « etwa wurde auch über den Einsatz von green helmets in Analogie zu UN-Blauhelmen nachgedacht, um

16 Der englische Begriff lautet »securitization«. Vgl. etwa Ole Waever, »Securitization and Desecuritization «, in: Ronnie D. Lipschutz, (Hrsg.), On Security, New York 1995, S. 46-86. 
Schaubild 1: Gegenüberstellung unterschiedlicher Sicherheitsbegriffe

\begin{tabular}{|c|c|c|c|}
\hline & $\begin{array}{c}\text { Klassischer Sicherheits- } \\
\text { begriff }\end{array}$ & $\begin{array}{c}\text { Erweiterter Sicherheits- } \\
\text { begriff }\end{array}$ & Human Security \\
\hline $\begin{array}{l}\text { Verständnis von Si- } \\
\text { cherheit }\end{array}$ & $\begin{array}{l}\text { nationale Sicherheit im Sinne } \\
\text { des Schutzes staatlicher territo- } \\
\text { rialer Integrität }\end{array}$ & $\begin{array}{l}\text { im Mittelpunkt weiterhin na- } \\
\text { tionale Sicherheit, lediglich } \\
\text { breiteres Verständnis möglicher } \\
\text { Sicherheitsbedrohungen }\end{array}$ & $\begin{array}{l}\text { komplementär zu staatli- } \\
\text { cher/nationaler Sicherheit, Fo- } \\
\text { kus auf den »menschlichen } \\
\text { Aspekten« von Sicherheit, } \\
\text { Rechten und Entwicklung }\end{array}$ \\
\hline Referenzobjekt & Staat & Staat & Individuum \\
\hline Art der Bedrohung & $\begin{array}{l}\text { (zwischenstaatliche) militäri- } \\
\text { sche Bedrohung }\end{array}$ & $\begin{array}{l}\text { neue Bedrohungslagen v.a. in } \\
\text { den Bereichen Umwelt, Wirt- } \\
\text { schaft, durch innerstaatliche } \\
\text { Konflikte, Terrorismus }\end{array}$ & $\begin{array}{l}\text { zwischen- und innerstaatliche } \\
\text { Konflikte, Migration, Post- } \\
\text { Konflikt-Situationen, Armut, } \\
\text { Gesundheitsbedrohungen }\end{array}$ \\
\hline Quelle der Bedrohung & Sicherheitsdilemma & $\begin{array}{l}\text { durch Auflösung der alten bi- } \\
\text { polaren Struktur Aufbrechen } \\
\text { alter und neuer Konflikte, } \\
\text { grenzüberschreitende Problem- } \\
\text { lagen }\end{array}$ & $\begin{array}{l}\text { sich im Zuge der Globalisie- } \\
\text { rung verstärkende Interdepen- } \\
\text { denzen, politische und öko- } \\
\text { nomische Ungleichheiten und } \\
\text { Instabilitäten }\end{array}$ \\
\hline $\begin{array}{l}\text { Mittel zur Abwehr der } \\
\text { Bedrohung }\end{array}$ & $\begin{array}{l}\text { Einsatz staatlicher Gewalt (vor } \\
\text { allem Militär) }\end{array}$ & $\begin{array}{l}\text { Einsatz staatlicher Gewalt, die } \\
\text { sich den veränderten Bedro- } \\
\text { hungsszenarien anpassen } \\
\text { muss, Erhöhung staatlicher } \\
\text { Handlungskapazität durch } \\
\text { verstärkte internationale Zu- } \\
\text { sammenarbeit }\end{array}$ & $\begin{array}{l}\text { Gegenmaßnahmen auf allen } \\
\text { Ebenen durch verschiedene Ak- } \\
\text { teure (Staaten, internationale } \\
\text { Organisationen, privat- } \\
\text { wirtschaftliche Akteure, zivil- } \\
\text { gesellschaftliche Akteure), } \\
\text { Schutz und Empowerment von } \\
\text { Betroffenen }\end{array}$ \\
\hline
\end{tabular}

im Falle von Ressourcenkonflikten militärisch eingreifen zu können. ${ }^{17}$

Eine Intention, die mit der Versicherheitlichungsstrategie einherging, war es ursprünglich, den Aufmerksamkeitswert für bislang vernachlässigte Themen zu erhöhen. Nun stellt sich allerdings beim Begriff der menschlichen Sicherheit das Problem einer großen begrifflichen Unschärfe. Es gibt keine klare Definition, lediglich eine lange Liste von Bereichen, die menschliche Sicherheit tangieren, wodurch der Begriff nahezu allumfassend wird. Durch die Fülle der Themen, die unter dem Stichwort »menschliche Sicherheit « bearbeitet werden sollen, ist damit letztendlich auch keine klare Prioritätensetzung mehr gegeben, wie es beim traditionellen Sicherheitsbegriff noch der Fall war. Somit wird das strategische Ziel verfehlt, traditionellen Entwicklungsthemen einen höheren Platz auf der politischen Tagesordnung zu verschaffen. Die Verteilungskämpfe um knappe Ressourcen sind die alten geblieben.

17 Vgl. Andronico O. Adede, "Management of Environmental Disputes: Avoidance versus Settlement «, in: Winfried Lang (Hrsg.), Sustainable Development and International Law, London 1995, S. 115-124.

\section{Sicherheit, Entwicklung, Rechte}

Wie kann in Anbetracht der geschilderten Nachteile das Konzept von menschlicher Sicherheit dennoch produktiv auch im Sinne der Forderungen nach mehr Geschlechtergleichheit - genutzt werden? Grundsätzlich bleibt festzuhalten, dass Sicherheit ein soziales Konstrukt ist, das sich in gesellschaftlichen Diskursen herausbildet und in und durch diese verändert werden kann. Mit dem Konzept von menschlicher Sicherheit besteht die Chance, die Gefährdungen, denen sich Individuen durch staatliche und nicht-staatliche Gewalt oder durch die Abwesenheit staatlicher Handlungsfähigkeit ausgesetzt sehen, nicht nur analytisch in den Blick zu bekommen, sondern diese auch politisch $\mathrm{zu}$ thematisieren. Diese Chance wäre jedoch vertan, wenn unterschiedliche Handlungslogiken, die aus anderen Perspektiven zum Wohlergehen und dem Schutz der physischen Unversehrtheit von Individuen beitragen, der Handlungslogik von »Sicherheit« untergeordnet würden oder in dieser aufgehen würden.

Der sicherheitspolitische Diskurs ist durch andere Machtstrukturen und Handlungslogiken geprägt als etwa der Entwicklungs- oder Menschenrechtsdiskurs. Themen, die im Sicherheitsdiskurs diskutiert werden, müssen sich immer einer Logik (und Prioritätensetzung) unterwerfen, die von den staatlichen Sicherheitsinteressen machtvoller Akteure bestimmt wird, wie die derzeitige Anti-Terrorismuspolitik 
drastisch zeigt. Im Sicherheitsdiskurs steht der Schutz der physischen und psychischen Unversehrtheit von Menschen und die Abwehr möglicher Bedrohungen - auch für staatliche territoriale Integrität - im Vordergrund. Im Entwicklungsdiskurs dagegen geht es vorrangig darum, das physische Überleben und das Wohlergehen von Menschen sicherzustellen sowie deren geistige und körperliche Entfaltungsmöglichkeiten $\mathrm{zu}$ fördern. Im Diskurs über Rechte wiederum werden Rechte und Pflichten formuliert, durch die ein handelndes Subjekt erst konstituiert wird und durch die dessen Handlungsbefugnis im Hinblick auf andere Rechtssubjekte begrenzt wird. Außerdem bestehen in den einzelnen Diskursen jeweils andere Vorstellungen über die Rolle von Individuen. Aus einer Sicherheitsperspektive wird die handelnde Person immer zur passiven Empfängerin von Schutz. Die Tatsache, dass ein Hauptmerkmal des Konzepts von Human Security darin gesehen wird, dass sich der Blick »from the state to the individual (and to particularly vulnerable groups such as women and children) « ${ }^{18}$ richtet, macht dieses Verständnis der besonders »verwundbaren « Gruppen, die nun geschützt werden sollen, sehr deutlich. Dieses Verständnis ist legitim, trägt aber nichts dazu bei, Geschlechterungleichheiten abzubauen. Aus einer Entwicklungsperspektive geht das Akteursverständnis einen Schritt weiter, denn im Entwicklungsdiskurs steht die Aktivierung individueller Entfaltungsmöglichkeiten - mit dem Schlagwort Empowerment versehen - im Vordergrund. Allein aus einer Rechtsperspektive wird das Individuum jedoch erst zu einem handlungsfähigen Subjekt mit einklagbaren Rechten und Pflichten, wie das Ringen um die Abschaffung der Sklaverei oder die Einführung des Wahlrechts für Personengruppen jenseits des »weißen Mannes « zeigt.

Die letztgenannten Beispiele verdeutlichen bereits, dass die Wirkungsmächtigkeit dieser unterschiedlichen Akteursverständnisse nicht trivial ist, weshalb eine Verengung des Begriffs von menschlicher Sicherheit auf den Aspekt des Schutzes der körperlichen und geistigen Unversehrtheit dringend notwendig scheint. Anstatt Entwicklung und Rechte unter das Dach von menschlicher Sicherheit zu stellen, sollten diese Bereiche gleichberechtigt als weitere Säulen einer außen- und sicherheitspolitischen Strategie verstanden werden, in der auch Geschlechterfragen Berücksichtigung finden können. ${ }^{19}$ Dies kann beispielsweise dadurch geleistet werden, dass über alle Bereiche hinweg eine Aufhebung der Trennung von öffentlicher und privater Sphäre angestrebt wird. Dadurch geraten auch im Sicherheitsbereich geschlechterspezifische Formen psychischer und physischer Bedrohungen wie Massenvergewaltigungen, ${ }^{20}$ häusliche Gewalt, Menschenhandel oder Zwangsprostitution in den Blickpunkt, die nur unzureichend mit militärischen Mitteln

18 S. Neil MacFarlane, »A Useful Concept that Risks Losing Its Political Salience«, in: Security Dialogue (3) 2004, S. 368-369, hier S. 368.

$19 \mathrm{Zu}$ einer ähnlichen Einschätzung gelangt Claudia v. Braunmühl bezogen auf den unabhängigen Stellenwert von Menschenrechten. Siehe Claudia v. Braunmühl, »Sicherheit für wen und wovor? Kritische Anfragen zum Sicherheitskonzept der Vereinten Nationen «, in: epd-Entwicklungspolitik, (1) 2002, S. 44-49.

20 Systematische Vergewaltigungen als Mittel in gewaltsamen Auseinandersetzungen betreffen übrigens sowohl Frauen als auch Männer, jedoch in unterschiedlichem (dokumentierten) Ausmaß und von der Intention der Vergewaltiger her gesehen mit unterschiedlichem Bedeutungsgehalt. abgewehrt werden können und eher eine Bearbeitung durch nicht-militärische Mittel notwendig machen. Ironischerweise hat insbesondere der Anstieg von Frauenhandel und Zwangsprostitution in Post-Konflikt-Gesellschaften wie im Kosovo deutlich gemacht, dass die Anwesenheit von Militär (namentlich der Friedenstruppe KFOR) und von Angehörigen der internationalen Übergangsverwaltung UNMIK ursächlich für diese Form der Bedrohung menschlicher Sicherheit ist. ${ }^{21}$ Ein weiterer sicherheitsrelevanter Bereich wäre aus der Perspektive von Human Security im engeren Sinne weiterhin die Bedrohung der Zivilbevölkerung durch bestimmte Waffentechnologien, worunter exemplarisch die Diskussion um eine Kleinwaffen-Konvention fällt.

Im Entwicklungsbereich stehen die gleichberechtigte Teilhabe und das Empowerment benachteiligter Akteursgruppen im Mittelpunkt, wodurch auch die Entwicklungsbedürfnisse von Frauen stärker Berücksichtigung finden. Dabei geht es um die Anerkennung von Frauen als ökonomische Subjekte, etwa was die Bedeutung von Hausarbeit anlangt, oder die konkrete Ausrichtung entwicklungspolitischer Projekte auf Frauen. Die Rechteperspektive schließlich führt dazu, dass Frauen als gleichberechtigte Rechtssubjekte anerkannt werden, deren spezifische »Rechtsansprüche « dann in den Vordergrund rücken. Insbesondere in diesem Bereich verzeichneten internationale Frauen-NGOs seit Beginn der 1990er Jahre signifikante Erfolge, beispielsweise bei der Thematisierung von häuslicher Gewalt, von Vergewaltigung als Straftatbestand im Statut des Internationalen Strafgerichtshofs oder bei der Einforderung »reproduktiver Rechte .

Die genannten konzeptuellen Unterschiede zwischen dem Sicherheits-, Entwicklungs- und Rechtsdiskurs sind nicht nur intellektuelle Spielereien, sie haben auch realpolitische Konsequenzen. In der Diskussion um die praktische Relevanz des Konzepts von Human Security verweisen einzelne AutorInnen immer wieder auf die Ottawa-Konvention, in der 1997 das Verbot von Antipersonen-Landminen festgeschrieben wurde, als einen der großen Erfolge des veränderten Sicherheitsverständnisses. Bei genauerer Betrachtung erweist sich jedoch gerade dieser Fall als paradigmatisch für den Einfluss der oben skizzierten Wirkungsmächtigkeit unterschiedlicher Diskurse und der mit ihnen verbundenen Arenen. So wurde beispielsweise jahrelang im Rahmen von UN-Abrüstungskonferenzen auch über das Verbot von Antipersonen-Landminen verhandelt, ohne ein konkretes Ergebnis zu erzielen. Eine Lösung konnte erst dann erreicht werden, als dieser Verhandlungsgegenstand auf Betreiben einer Koalition von Nichtregierungsorganisationen und unter der Schirmherrschaft Kanadas räumlich und organisatorisch aus dem Sicherheitskontext der UN-Abrüstungsverhandlun-

21 Darauf wurde in den letzten Jahren immer wieder in diversen Berichten der Sonderberichterstatterin der UN-Menschenrechtskommission zu Gewalt gegen Frauen hingewiesen (http://www.unhchr.ch/html/ menu2/7/b/ mwom.htm). Sehr dezidiert nimmt dazu ein Bericht von Amnesty International Stellung. Siehe Amnesty International: "So does that mean I have rights? « Protecting the human rights of women and girls trafficked for forced prostitution in Kosovo (Report 6 May 2004), London 2004 (http://web.amnesty.org/library/Index/ ENGEUR700102004?open\&of=ENG-YUG). 
Schaubild 2: Drei Säulen einer geschlechtergerechten Außen- und Sicherheitspolitik

\begin{tabular}{|c|c|c|c|}
\hline & $\begin{array}{l}\text { Human } \\
\text { SECURITY }\end{array}$ & $\begin{array}{l}\text { Human } \\
\text { DEVELOPMENT }\end{array}$ & $\begin{array}{l}\text { Human } \\
\text { RIGHTS }\end{array}$ \\
\hline $\begin{array}{l}\text { Zugrundeliegende } \\
\text { Handlungslogik }\end{array}$ & $\begin{array}{l}\text { Schutz der psychischen und } \\
\text { physischen Unversehrtheit, } \\
\text { Abwehr von Bedrohungen }\end{array}$ & $\begin{array}{l}\text { Sicherstellung des physischen } \\
\text { Überlebens und Wohlerge- } \\
\text { hens, Förderung der geistigen } \\
\text { und körperlichen Ent- } \\
\text { faltungsmöglichkeiten }\end{array}$ & $\begin{array}{l}\text { Zuweisung von Rechten und } \\
\text { Pflichten, die ein handelndes } \\
\text { Subjekt konstituieren und des- } \\
\text { sen Handlungsbefugnis im } \\
\text { Hinblick auf andere Rechts- } \\
\text { subjekte begrenzen }\end{array}$ \\
\hline Akteursverständnis & $\begin{array}{l}\text { passive EmpfängerIn von } \\
\text { Schutz }\end{array}$ & $\begin{array}{l}\text { Aktivierung individueller } \\
\text { Handlungsfähigkeit }\end{array}$ & $\begin{array}{l}\text { handlungsfähiges Subjekt mit } \\
\text { einklagbaren Rechten und } \\
\text { Pflichten }\end{array}$ \\
\hline $\begin{array}{l}\text { Integration der } \\
\text { Geschlechter- } \\
\text { perspektive durch... }\end{array}$ & $\begin{array}{l}\text { Aufhebung der Trennung von } \\
\text { öffentlicher und privater Sphä- } \\
\text { re; Wahrnehmung geschlech- } \\
\text { terspezifischer Formen psychi- } \\
\text { scher und physischer } \\
\text { Bedrohungen und deren Ab- } \\
\text { wehr unter Einsatz militäri- } \\
\text { scher und nicht-militärischer } \\
\text { Mittel }\end{array}$ & $\begin{array}{l}\text { Aufhebung der Trennung von } \\
\text { öffentlicher und privater Sphä- } \\
\text { re; Gewährung von gleichbe- } \\
\text { rechtigten Teilhabemöglich- } \\
\text { keiten; Empowerment } \\
\text { benachteiligter Akteure; Be- } \\
\text { rücksichtigung der Entwick- } \\
\text { lungsbedürfnisse von Frauen }\end{array}$ & $\begin{array}{l}\text { Aufhebung der Trennung von } \\
\text { öffentlicher und privater Sphä- } \\
\text { re; Anerkennung von Frauen } \\
\text { als gleichberechtigten Rechts- } \\
\text { subjekten; Berücksichtigung } \\
\text { der spezifischen »Rechtsan- } \\
\text { sprüche« von Frauen }\end{array}$ \\
\hline
\end{tabular}

gen ausgegliedert wurde. Damit eröffnete sich auch für eine Reihe nicht-staatlicher Akteure wie die International Campaign to Ban Landmines (ICBL), die im traditionellen UNAbrüstungskontext bislang weniger Gehör gefunden hatten, ein besserer Zugang zum politischen Entscheidungsprozess. Gleichzeitig konnte in einer Studie, die das Internationale Komitee vom Roten Kreuz (IKRK) in Auftrag gegeben hatte, gezeigt werden, dass Entwicklungschancen von Gesellschaften durch zahlreiche menschliche Opfer von Landminen auch nach Abschluss von Kampfhandlungen - behindert werden. ${ }^{22}$ Letztendlich, so die Argumentation der BefürworterInnen eines Verbots von Antipersonen-Landminen, sei der »ökonomische Schaden« viel größer als der vermeintliche militärische Nutzen. Ihre Strategie bestand darin, das Thema aus dem Sicherheitsdiskurs in den Entwicklungsdiskurs zu überführen. ${ }^{23}$ Dieses Beispiel zeigt anschaulich, wie die inhaltliche Einordnung eines Gegenstandes in eine bestimmte Thematik einzelne Handlungsmöglichkeiten verschließt oder eröffnet, je nachdem, innerhalb welcher Diskurse Themen diskutiert werden und welche Akteure die dominierende Handlungslogik vorgeben.

22 ICRC, Anti-Personnel Landmines: Friend or Foe? A Study on the Military Use and Effectiveness of Anti-Personnel Mines, Genf 1996.

23 Vgl. Cornelia Ulbert/Simone Wisotzki, »(Pre-) Structuring Argumentative Processes: The Impact of Institutional Settings on Banning Worst Forms of Child Labour and Landmines «, Paper Presented at the 4th Pan European International Relations Conference, University of Kent, Canterbury, Sept. 8-10, 2001.

\section{Fazit: Weniger ist mehr}

Human Security ist ein Konzept, das durch den Fokus auf Individuen prinzipiell großes Potential zur Integration einer Geschlechterperspektive aufweist. In der Umsetzung des Konzeptes in tatsächliche Politik zeigen sich jedoch aufgrund der Breite und begrifflichen Unschärfe des Konzeptes eine Reihe von Schwierigkeiten, wie etwa auch die Bemühungen Kanadas zeigen, die eigene Außen- und Sicherheitspolitik daran zu orientieren. Die Strategie, Themen einen höheren Stellenwert auf der politischen Tagesordnung $\mathrm{zu}$ verschaffen, läuft in Anbetracht der allgemein diskutierten Breite ins Leere, wodurch auch der strategische Nutzen zur Durchsetzung geschlechterpolitischer Forderungen leidet. Hinzu kommen die negativen Effekte, die mit der »Versicherheitlichung « von Themen verbunden sind, nämlich die Tatsache, dass mit den »traditionellen « Akteuren des Sicherheitsbereichs machtvolle Akteure zu DiskursteilnehmerInnen werden, die mit ihrer Handlungslogik und ihren Prioritätensetzungen Diskurse dominieren können, und sich der Fokus dann häufig auf eine militärische Problembearbeitung hin verschiebt oder zumindest davon dominiert wird. Konzeptionell sinnvoller scheint daher - nicht nur aus einer Geschlechterperspektive - eine außen- und sicherheitspolitische Strategie, die auf den drei Säulen Sicherheit, Entwicklung und Rechte basiert mit einer deutlichen Einengung des Begriffs von menschlicher Sicherheit auf den Schutz der körperlichen und geistigen Unversehrtheit von Individuen. Diese inhaltliche Verengung und Fokussierung von menschlicher Sicherheit verspräche ein Mehr an politischer Wirksamkeit. 